\title{
MicroRNA-194 inhibits epithelial to mesenchymal transition of endometrial cancer cells by targeting oncogene BMI-1
}

\author{
Peixin Dong ${ }^{1}$, Masanori Kaneuchi ${ }^{1}$, Hidemichi Watari ${ }^{1}$, Junichi Hamada ${ }^{2}$, Satoko Sudo ${ }^{1}$, Jingfang Ju ${ }^{3}$ and \\ Noriaki Sakuragi ${ }^{1 *}$
}

\begin{abstract}
Background: Epithelial-mesenchymal transition (EMT) is the key process driving cancer metastasis. Oncogene/self renewal factor BMI-1 has been shown to induce EMT in cancer cells. Recent studies have implied that noncoding microRNAs (miRNAs) act as crucial modulators for EMT. The aims of this study was to determine the roles of BMI-1 in inducing EMT of endometrial cancer (EC) cells and the possible role of miRNA in controlling BMI-1 expression.

Methods and results: We evaluated the expression of BMI-1 gene in a panel of EC cell lines, and detected a strong association with invasive capability. Stable silencing of BMI-1 in invasive mesenchymal-type EC cells upregulated the epithelial marker E-cadherin, down-regulated mesenchymal marker Vimentin, and significantly reduced cell invasion in vitro. Furthermore, we discovered that the expression of BMI-1 was suppressed by miR-194 via direct binding to the BMI-1 3'-untranslated region 3'-UTR). Ectopic expression of miR-194 in EC cells induced a mesenchymal to epithelial transition (MET) by restoring E-cadherin, reducing Vimentin expression, and inhibiting cell invasion in vitro. Moreover, BMI-1 knockdown inhibited in vitro EC cell proliferation and clone growth, correlated with either increased p16 expression or decreased expression of stem cell and chemoresistance markers (SOX-2, KLF4 and MRP-1).
\end{abstract}

Conclusion: These findings demonstrate the novel mechanism for BMl-1 in contributing to EC cell invasion and that repression of BMI-1 by miR-194 could have a therapeutic potential to suppress EC metastasis.

\section{Introduction}

Endometrial cancer (EC) is the most frequent gynecologic malignancy in the developed countries [1]. Although the incidence of EC is lower in East Asian than in Western countries, it tends to increase markedly in recent years [2]. EC is generally classified as type I endometrioid EC or type II non endometrioid EC based on etiology and clinical variables. The majority of EC are of type I, which are associated with good prognosis. However, myometrial invasion and distant metastasis decreases the survival rates of patients after surgical treatment. In contrast, type II EC is often related to poor prognostic factors, such as high grade or deep myometrial penetration. Thus, to further improve

\footnotetext{
* Correspondence: sakuragi@med.hokudai.ac.jp

'Department of Gynecology, Hokkaido University Graduate School of Medicine and School of Medicine, Hokkaido University, Sapporo, Japan Full list of author information is available at the end of the article
}

patient survival, it is essential to further understand the molecular and cellular mechanism of EC development, and in turn, to develop novel therapeutic strategies to block EC progression.

The epithelial to mesenchymal transition (EMT) is a key process contributing to cancer metastasis, characterized by the loss of the epithelial marker E-cadherin, an increase in the mesenchymal markers Vimentin and $\mathrm{N}$-cadherin, and an increase in the migratory and invasive behavior [3]. BMI-1 (B lymphoma mouse Moloney leukemia virus insertion region 1 ) is a self-renewal gene and overexpressed in multiple human cancers, including lung cancer [4], breast cancer [5], prostate cancer [6], ovarian cancer [7], and recently EC [8]. BMI-1 upregulation is associated with malignant transformation in hepatocellular carcinoma [9]. Notably, recent research has shown that BMI-1 plays essential roles in inducing EMT in head and neck squamous cell carcinoma [10]. However, the roles of BMI-

\section{C) Biomed Central}


1 in EC metastasis and the molecular mechanism regulating BMI-1 expression remain to be investigated.

Epigenetic alterations (methylation, non-coding microRNA) are critical to cancer development [11]. MicroRNAs (miRNAs) are regulatory, single-stranded noncoding RNAs that repress protein expression by basepairing with the 3' untranslated region (UTR) of the target mRNA, which triggers either mRNA translation repression or RNA degradation [12]. Aberrant levels of miRNA have been reported in a variety of human cancers, including EC [13]. These observations promote us to hypothesize that certain miRNA may control BMI-1 expression in EC cells, and thus have a therapeutic potential against EC cancer progression.

In this study, we provide experimental evidence that BMI-1 is essential for EMT and invasive phenotype in EC cells. We discovered a novel post-transcriptional regulatory mechanism of BMI-1 expression mediated by miR-194 by directly interacting with the BMI-1 mRNA at the 3'-UTR. The expression of BMI-1 protein level was suppressed by miR-194 with MET transition associated with reduced EC tumor invasion. As a result, it provides a potential new strategy to prevent EC progression by targeting oncogene BMI-1.

\section{Materials and methods \\ Cell lines}

Human EC cell lines HHUA (well differentiated), HOUA-I (moderately differentiated) and HEC-50B (poorly differentiated) were obtained from RIKEN cell bank (Tsukuba, Japan) and grown in Minimum Essential Medium Eagle (Sigma-Aldrich, UK) supplemented with $15 \%$ of fetal bovine serum (Cambrex Bioscience, Belgium). The cells were maintained at $37^{\circ} \mathrm{C}$ in a humidified atmosphere of $5 \% \mathrm{CO}_{2}$.

\section{Selection of invasive EC cells in transwell invasion chamber}

Subpopulations from HEC-50B cells were selected as described previously [14], 6 using transwell plates. Briefly, the polycarbonate membranes (containing $8-\mu \mathrm{m}$ pores) of the transwell inserts were coated with Matrigel gel (BD Biosciences, CA). Cells were resuspended in serum-free MEM and seeded into the upper wells. MEM medium supplemented with $15 \%$ bovine serum was placed into the lower chamber. Following incubation for $24 \mathrm{~h}$ at $37^{\circ} \mathrm{C}$, the inserts were removed. The cells that migrated through the membranes and attached to the lower-chamber compartments were harvested aseptically and named as HEC-50B-highly invasive (HI)

\section{Western blot analysis}

Whole cellular protein was obtained with M-Per Mammalian Protein Extraction Reagent (Pierce Biotechnology,
MA). The aliquots were separated on SDS-PAGE (10\%) and transferred to nitrocellulose membranes. Antigenantibody complexes were detected ECL blotting analysis system (Amersham Pharmacia Biotech, UK). The BMI-1 antibody (ab38295) was purchased from Abcam (Cambridge, MA). E-cadherin antibody (A01589) and Vimentin antibody (A01189) were obtained from GenScript (Edison, NJ). Antibody for p16 (sc-468) and GAPDH (sc47724) were purchased from Santa Cruz Biotechnology (Santa Cruz, CA).

\section{Enforced miR-194 expression}

HEC-50B-HI cells were transfected with $50 \mathrm{nM}$ of precursor miR-194, or negative control precursor miRNA (Ambion, Austin, TX) by use of Lipofectamine 2000 (Invitrogen, Carlsbad, CA). After $48 \mathrm{~h}$, the cells were processed for Western blot, invasion assay, proliferation, or colony formation assay.

\section{In vitro cell invasion assay}

HEC-50B-HI transfected with precursor miR-194, or negative control precursor miRNA were harvested $48 \mathrm{~h}$ after transfection and re-suspended in serum-free MEM. $2 \times 10^{4}$ cells in $500 \mu \mathrm{l}$ of MEM medium were added into the upper chamber. In the lower chamber, $750 \mu \mathrm{l}$ of MEM medium containing $15 \%$ fetal bovine serum and $10 \mu \mathrm{g} / \mathrm{ml}$ of bovine fibronectin (Invitrogen, Germany) were placed. The cells were allowed to migrate through the intermediate membrane for $24 \mathrm{~h}$ at $37^{\circ} \mathrm{C}$. Membranes were then fixed with $10 \%$ neutral-buffered formalin and stained in $10 \%$ Giemsa solution. The cells attached to the lower side of the membrane were counted in ten high-powered $(200 \times)$ fields under a microscope. Assays were done in triplicate for each experiment, and each experiment was repeated three times.

\section{MiRNA quantitative real-time reverse transcription PCR}

Total RNA containing small RNA was extracted from cell lines by TRIzol reagent (Invitrogen, CA) according to the manufacturer's instructions. Quantitative reverse transcription (qRT)-PCR was performed to detect the levels of hsa-miR-194 in three EC cell lines by NCode miRNA qRT-PCR Analysis (Invitrogen, CA) according to the manufacturer's protocol. A qRT-PCR forward primer for hsa-miR-194 (5'-TGTAACAGCAACTCCATGTGG-3') was designed and synthesized by Invitrogen. GAPDH was used for normalization [15]. All real-time PCR assays were conducted as previously described [16].

\section{Luciferase activity assay}

The 3'-UTR vector of BMI-1 containing an intact miR194 recognition sequence was purchased from OriGene 
Technologies (Rockville, MD). A pGL3 construct containing BMI-1 3'-UTR with point mutations in seed sequence was constructed using a QuickChange sitedirected mutagenesis kit (Stratagene, CA), using the following primers: 5'-CATTACTTTTACATATATTGCT GGCCCTTCTGCTTTC-3' (forward) and 5'-GAAAGCAGAAGGGCCAGCAATATATGTAAAAGTAATG-3' (reverse). Cells were transfected with $50 \mathrm{nM}$ precursor miRNA (miR-194, miR-128 or control miRNA) along with the wild-type or mutant BMI-1 3'-UTR-luciferase constructs. 24 hours after transfection, luciferase activity was measured using dual-luciferase assay (Promega, Madison, WI).

\section{BMI-1 silencing by shRNA}

BMI-1 shRNA plasmids (sc-29814-SH) and control shRNA plasmids (sc-108060) were obtained from Santa Cruz Biotechnology (Santa Cruz, CA). HEC-50B-HI cells were stably transfected as described previously [17]. In brief, cells at $70 \%$ confluency were transfected with Lipofectamine PLUS Reagent (Invitrogen, CA) according to the manufacturer's protocols and selected in MEM medium containing puromycin (Sigma-Aldrich, MO) at $1 \mu \mathrm{g} / \mathrm{ml} 48 \mathrm{~h}$ post-transfection. Selected clones of HEC50B-HI cells were expanded into HEC-50B-HI-BMI-1 shRNA cells and HEC-50B-HI control shRNA cells, respectively.

\section{MTT assay}

For measurements of cell proliferation rates, $1 \times 10^{3}$ cells were plated into each well of 96-well plates and cultured in $100 \mu \mathrm{l}$ of medium containing $10 \%$ serum. After 1 or 5 days incubation, $10 \mu \mathrm{l}$ of MTT solution (Cell counting kit-8, Dojindo, Japan) 10 was added into each well, and plates were incubated for $4 \mathrm{~h}$ at $37^{\circ} \mathrm{C}$, and $450 \mathrm{~nm}$ UV absorbance of each sample was measured in a microplate reader. Assay was done in triplicate wells, and each experiment was repeated three times.

\section{Colony formation assay}

About 500 cells were seeded per well in 6-well plates. After $10 \mathrm{~d}$, the cells were fixed in 70\% ethanol and stained with $10 \%$ Giemsa (Sigma- Aldrich, MO). Colonies consisting of $>50$ cells were counted. The results represented the average of three independent experiments.

qRT-PCR of stemness markers and chemoresistance genes Real-time RT-PCR was performed using a Primescript One Step RT-PCR Kit (Takara, Japan). Primers used for SOX-2, KLF4, MRP-1 and GAPDH were previously reported [18].

\section{Statistical analysis}

All experiments were performed in triplicates. Statistical analyses were performed using SPSS statistical software. Student's t-test was adopted. Significance was 11 defined as $P<0.05$.

\section{Results}

BMI-1 expression correlates with invasive potential and EMT phenotype of EC cells

BMI-1 is thought to mediate cell invasion in several types of cancer. To assess the role of BMI-1 in conferring invasive properties to EC cells, we used Western blot analysis to characterize expression of BMI-1 in four human EC-derived cell lines (HHUA, HOUA-I, HEC50B and HEC-50B-HI), and determined whether expression of endogenous BMI-1 correlated with invasive ability by using an in vitro cell invasion assay. In contrast to HHUA cells with markedly reduced endogenous BMI-1 expression, HEC-50B-HI cells expressing 5-fold higher levels of BMI-1 protein exhibited the highest invasive potential after $24 \mathrm{~h}$ incubation (Figure 1A). The number of HEC-50B-HI cells that passed through the membrane was 5.0 times larger than the number of HHUA cells (Figure 1B), thus suggesting that BMI-1 expression level seemed to be closely associated with the enhanced invasive activities of EC cell lines.

It has been suggested that EMT plays a crucial role in cancer metastasis [3]. 12 During the acquisition of EMT characteristics, cancer cells lose the intercellular junctions and gain fibroblast-like motile and invasive phenotype, associated with the down-regulation of epithelial marker E-cadherin and the up-regulation of mesenchymal marker Vimentin [3]. To test the hypothesis that BMI-1 would be involved in the acquisition of an invasive EMT phenotype in EC cells, we evaluated the morphological changes between HEC-50B-derived aggressive sub-cell lines and its parental HEC-50B cells. Highly invasive HEC-50B-HI cells displayed spindle-like, fibroblastic morphology when comparing to HEC-50B cells showing epithelial-like appearance (Figure 1C). In accordance with this finding, expression of E-cadherin was significantly reduced in HEC-50B-HI cells. In contrast, expression of Vimentin was significantly increased in this cell line (Figure 1D), demonstrating that up-regulation of BMI-1 may contribute to EMT-derived invasive phenotype in EC cells.

\section{Silencing of BMI-1 expression reverts EMT phenotype and reduces $\mathrm{EC}$ cell invasion}

To validate whether BMI-1 affects EMT and cell invasion of EC cells, HEC-50B- BMI-1 shRNA and control shRNA cell lines were established. The BMI-1 protein level was significantly decreased in BMI-1 shRNA- 


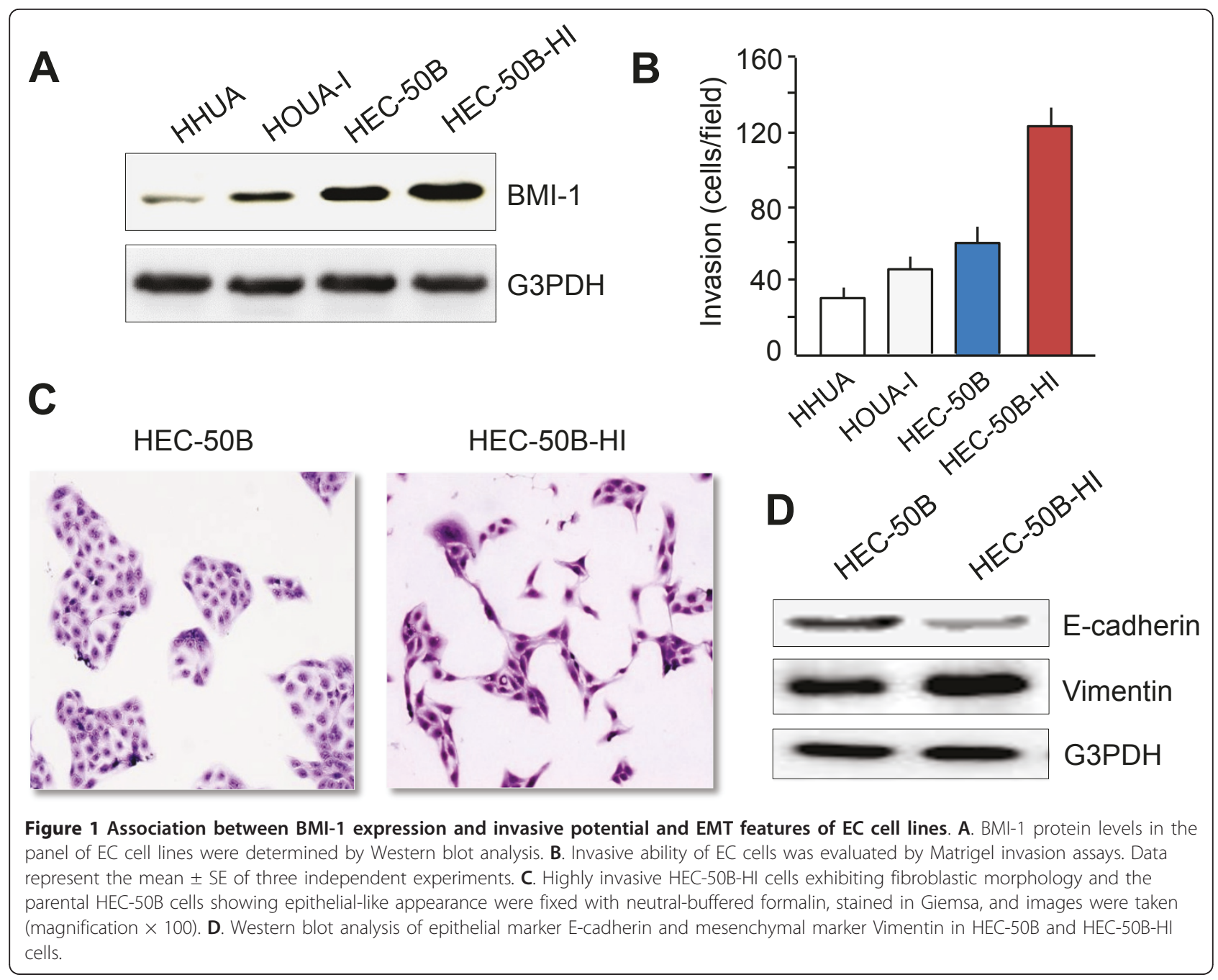

transfected HEC-50B-HI cells (Figure 2A). Knockdown of BMI-1 protein seemed to change the morphology from spread fibroblastoid to epithelial-like appearance (Figure 2B), decreased cell invasion (Figure 2C), and resulted in the up-regulation of E-cadherin and downregulation of Vimentin at protein levels (Figure 2D). These results suggest that BMI-1 is critical for the acquisition of EMT characteristics in EC cells.

\section{MiR-194 directly targets BMI-1, and reverses EMT phenotype in EC cells}

Based on miR target analysis using TargetScan website [19], miR-194 was identified as potential regulator of BMI-1 expression. The predicted binding of miR-194 with BMI-1 3'UTR was illustrated (Figure 3A), indicating that miR-194 is a potential miRNA targeting BMI-1. To compare the relationship between miR-194 and BMI-1 expression, we examined the endogenous miR194 expression level by qRT-PCR in HEC-50B-HI and HEC-50B cells. MiR-194 mRNA expression was significantly lower in high BMI-1 expressing HEC-50BHI cells (Figure 3B), showing that miR-194 level inversely correlated with BMI-1 expression. To examine the inhibitory effect of miR-194 on BMI-1 protein level, we performed Western blot analysis at $48 \mathrm{~h}$ after miR-194 transfection into HEC-50B-HI and HHUA cells. Ectopic expression of miR-194 in these two cell lines significantly decreased the protein levels of BMI-1 compared to 14 control miRNA (Figure 3C).

To further confirm that BMI-1 is the direct target of miR-194, firefly luciferase reporter vector containing a segment of the 3'UTR of BMI-1 with point mutations in the seed sequence was constructed. In addition, miR128 has been reported to target BMI-1 in glioma [20]. Therefore miR-128 was used as a positive control. The BMI-1 luciferase constructs were then co-transfected with miR-194, miR-128 or control miRNA into HEC50B-HI as well as HHUA cells. As expected, transfection with miR-128 dramatically attenuated BMI-1 3'-UTR luciferase activity. Interestingly, the BMI-1 3'-UTR 


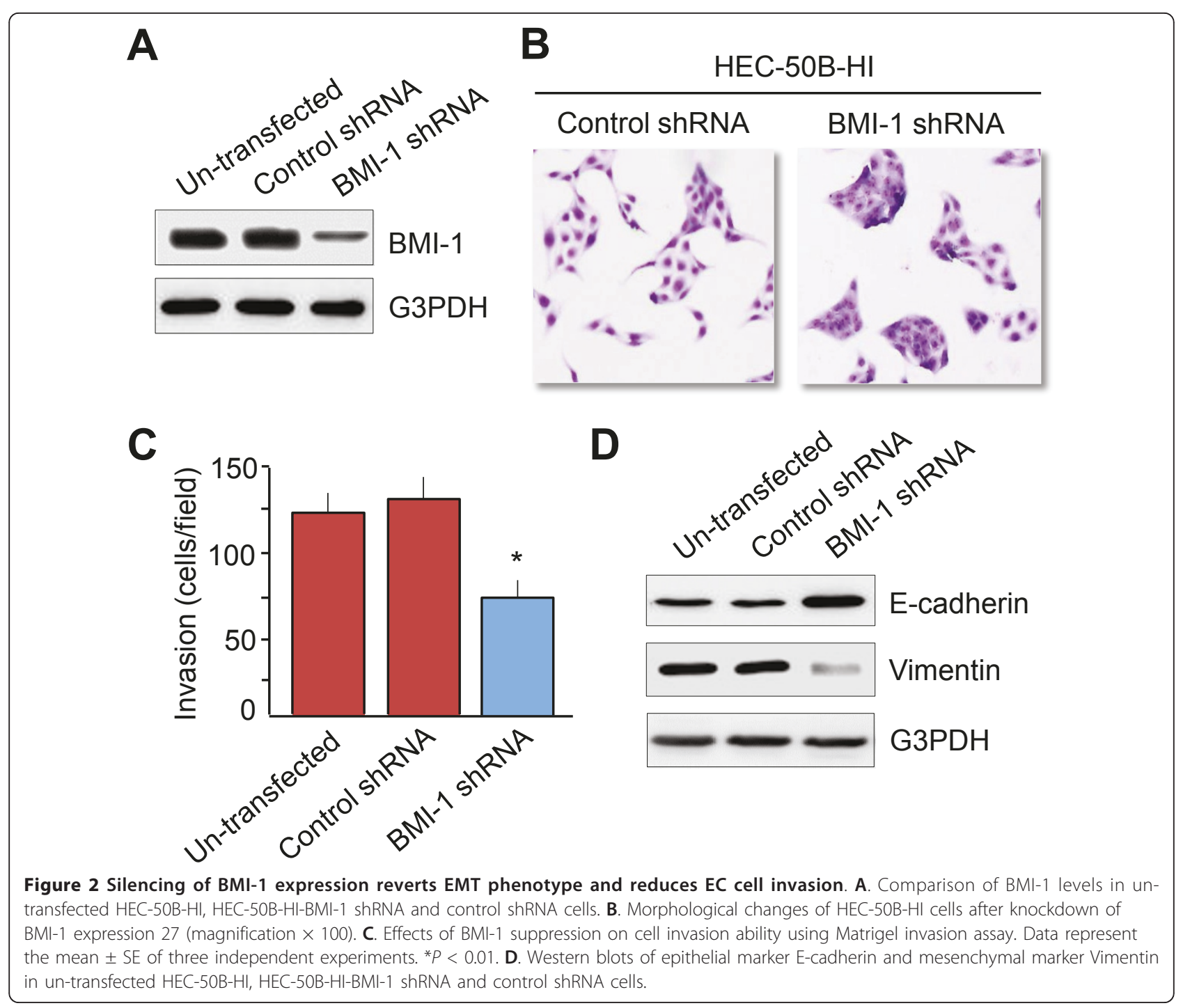

luciferase activity in miR-194-transfected both HEC50B-HI and HHUA cells was significantly lower when comparing with control miRNA-transfected cells, whereas mutation of miR-194 recognition site rescued the luciferase activity (Figure 3D). These results collectively suggested a direct and specific inhibition of miR194 on BMI-1 3'-UTR in EC cells.

BMI-1 has been shown to promoting EMT in head and neck squamous cell carcinoma [10], implies the possibility that reexpression of miR-194 may lead to a reversal of EMT phenotype of invasive EC cells. To test our hypothesis, the effect 15 of ectopic expression of miR-194 on cell invasion and expression of EMT markers was investigated in HEC-50B-HI and HHUA cells. Transfection with miR-194 significantly decreased cell invasion in these cells. However, this inhibition was more evident in HEC-50B-HI cells (Figure 3E). Furthermore, transfection of miR-194 induced a loss of the mesenchymal phenotype by restoring epithelial marker E-cadherin and reducing mesenchymal marker Vimentin expression (Figure 3C). These results indicated that reexpression of miR-194 could reverse the EMT phenotype, and dramatically decreased cell invasion in BMI-1 expressing EC cells.

\section{Knockdown of BMI-1 repressed in vitro cell proliferation and clonal growth}

It has been shown that BMI-1 is required for the clonogenic growth of multiple myeloma cells [21]. Therefore, we examined the roles of BMI-1 in regulating proliferation and colony growth of HEC-50B-HI cells. As expected, cell proliferation was significantly decreased in BMI-1 shRNA-transfected cells compared with control cells (Figure 4A). Moreover, colony formation of HEC50B-HI cells was significantly reduced after BMI-1 protein expression was effectively suppressed by shRNA 


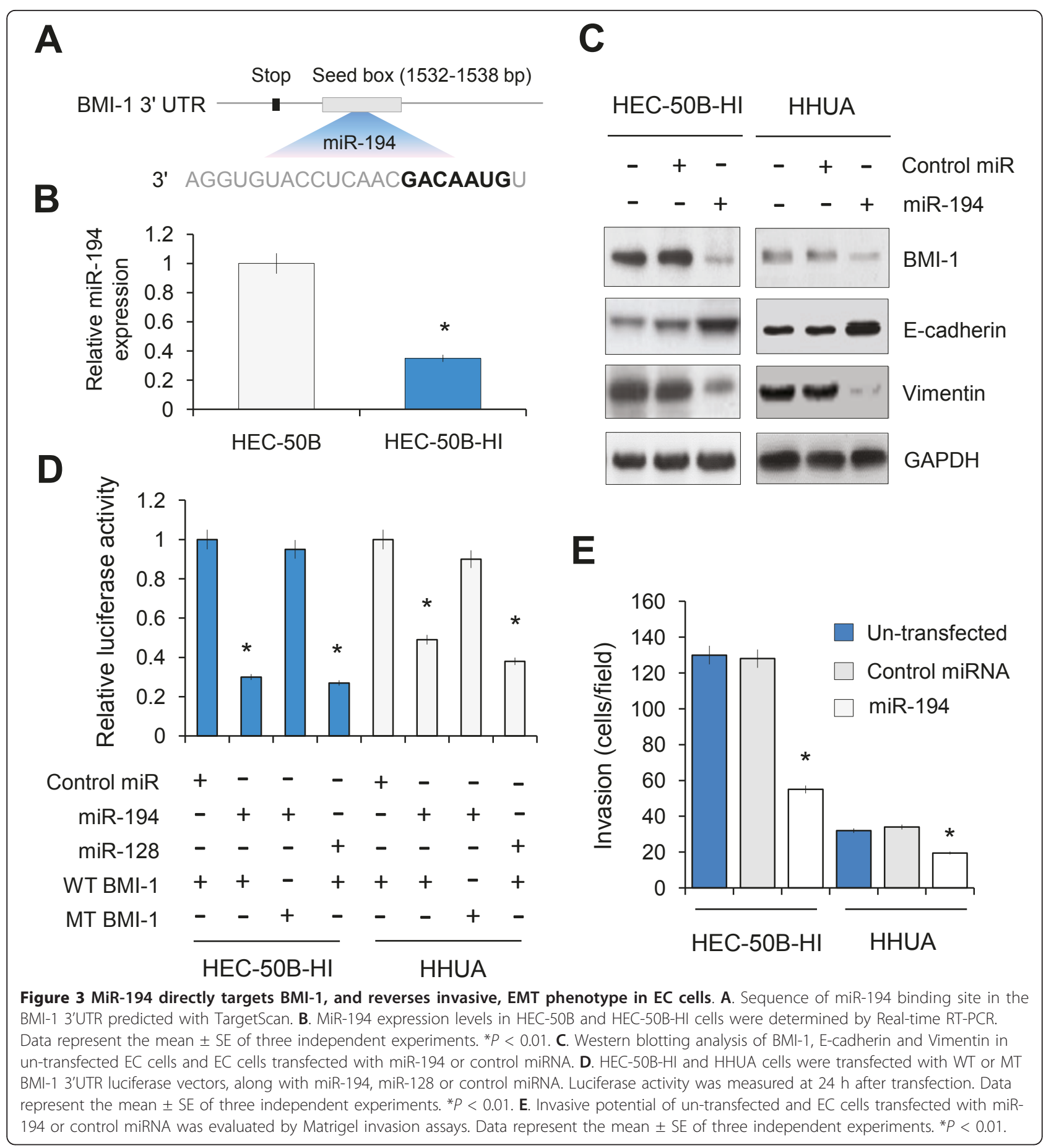

transfection (Figure 4B). Since previous studies have shown that BMI-1 promotes cell proliferation through suppression of tumor-suppressor p16 [22], we determined the p16 protein level by Western blot. Down-regulation of BMI-1 expression by BMI-1 shRNA transfection significantly increased p16 protein expression (Figure 4C). These results indicated that knockdown expression of BMI-1 could suppress EC cell proliferation and colony growth in EC cells by upregulation of p16 expression.

Overexpression of BMI-1 in head and neck squamous cell carcinoma promote stemness properties by increasing the expression of stem cell marker and drug-resistance gene [18]. By using qRT-PCRs, we demonstrated that the mRNA expression levels of stemness genes SOX-2, KLF4 and chemoresistance gene MRP-1 were 
A

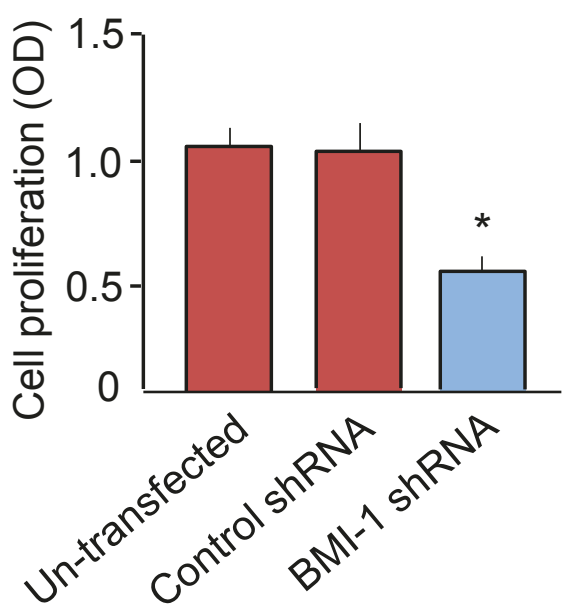

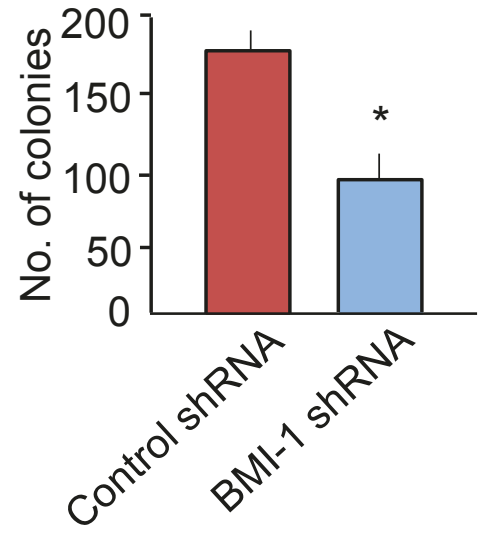

C

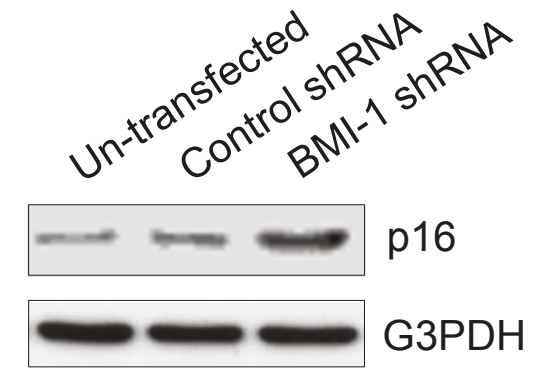

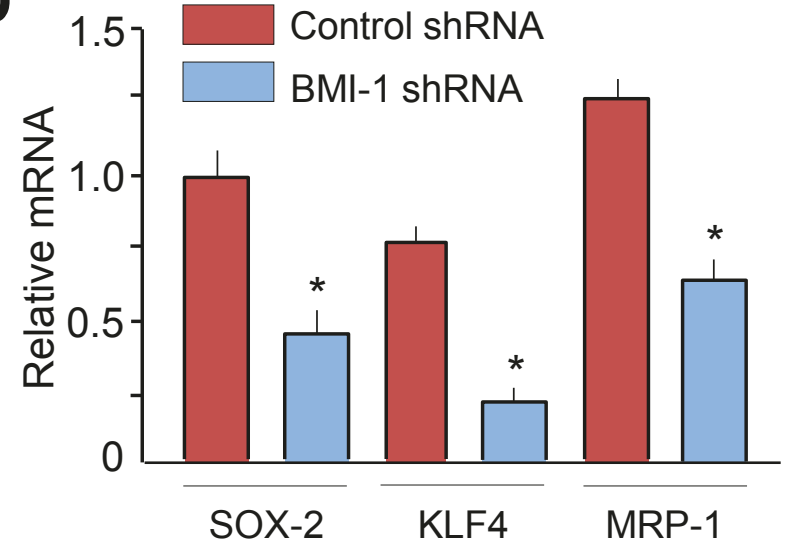

Figure 4 Knockdown of BMI-1 repressed in vitro cell proliferation and clone growth. A. Effects of BMI-1 repression on cell proliferation assessed by MTT assay in un-transfected HEC-50B-HI cells, HEC-50B-HI-BMI-1 shRNA and control shRNA cells. ${ }^{*} P<0.05$. B. Colony formation was assessed following stable repression of BMI-1. ${ }^{*} P<0.05$. C. Western immunoblot analysis of p16 protein expression in un-transfected HEC-50B-H cells, HEC-50B-HI-BMI-1 shRNA cells and control shRNA cells. D. mRNA expression levels of SOX-2, KLF4 and MRP-1 in HEC-50B-HI-BMI-1 shRNA and control shRNA cells were determined by Real-time RT-PCR. Data represent the mean \pm SE of three independent experiments.

downregulated in HEC-50B-HI-BMI-1 shRNA cells compared with control shRNA cells (Figure 4D). These results suggest the important roles of BMI-1 in modulating cell proliferation and stemness in EC.

\section{Discussion}

Previous studies have linked BMI-1 upregulation to enhanced cell invasion in various human tumors [4-7]. However, the role of BMI-1 in EC cell invasion has not been thoroughly explored. In this study, we discovered that elevated BMI-1 expression closely associated with EC cell invasion. In line with our study, up- regulation of BMI-1 has been shown to induce breast cancer metastasis [23]. Similarly, silencing of BMI-1 can inhibit the metastatic capability of cervical cancer cells [24]. Our results provided new evidence to support the oncogenic roles of BMI-1 in enhancing EC metastasis.

The acquisition of EMT-derived phenotype confers invasive behavior of tumor cells. BMI-1 is able to induce EMT in nasopharyngeal epithelial cells by repressing Ecadherin expression and up-regulating Vimentin expression [25]. We showed for the first time that knockdown of BMI-1 in EC cells led to an attenuation of in vitro invasion and a reversion of EMT, evidenced by epithelial-like morphology, increased expression of E-cadherin and decreased expression of Vimentin. These findings highlight the pivotal role for BMI-1 in driving invasive potential in EC cells through induction of EMT phenotype. 
Dysregulation of miRNAs are implicated in EMT modulation [26]. MiRNAs, such as miR-200 family and miR-205, act as key modulators of EMT and enforcers of the epithelial phenotype [27]. We showed that miR194 suppress BMI- 1 expression through direct interaction and inhibited EC cell invasion. These results were consistent with a recent report that miR-194 reduces invasion of the mesenchymal-18 like liver cancer cells both in vitro and in vivo [28]. The finding that BMI-1 expression was also reduced by overexpressing miR-194 in HHUA cells with low level of BMI-1 may reflect the heterogeneity of EC. Sub-populations of this cell line might be more aggressive and exhibit higher BMI-1 expression. Thus, targeting BMI-1 in invasive EC cells by miR-194 would be valuable in development of new therapeutic strategies against EC metastasis.

Cancer stem cell (CSC) has been demonstrated to play important roles in cancer metastasis [3]. In many CSC populations, aberrant BMI-1 expression is reported [29]. CSC isolated from EC has been shown to express BMI1 [30]. We demonstrated that knockdown of BMI-1 in invasive mesenchymal EC cells significantly reduced in cell proliferation, colony growth and up-regulated the p16 tumor suppressor. Many independent studies also support the association between BMI-1-dependent p16 suppression and enhanced stem cell proliferation [22]. Moreover, we demonstrated that loss of BMI-1 in EC cells reduces expression of stemness gene SOX-2, KLF4 and drug-resistance gene MRP-1, suggesting that BMI-1 expression maybe required for the proliferation of cancer cells, as well as for regulation of stemness properties of EC cells.

In conclusion, we discovered a critical function of BMI-1 to potentiate EC metastasis via induction of EMT. We also revealed a new regulatory mechanism of BMI-1 expression by miR-194 to inhibit EC cell invasion through down-regulation of BMI-1. As a result, restoration of miR-194 expression could have important implications for the clinical management of EC.

\footnotetext{
Acknowledgements

This work was partly supported by a Grant-in-Aid for Scientific Research (B) (No. 18390442) from Japan Society for the Promotion of Science (JSPS) of Japan and a Grant-in-Aid from the Ministry of Health, Labour and Welfare of Japan.

\section{Author details}

'Department of Gynecology, Hokkaido University Graduate School of Medicine and School of Medicine, Hokkaido University, Sapporo, Japan. ${ }^{2}$ Division of Cancer-Related Genes, Institute for Genetic Medicine, Hokkaido University, Sapporo, Japan. ${ }^{3}$ Department of Pathology, Stony Brook University Medical Center, NY, USA.
}

\section{Authors' contributions}

DP and JJ designed research; DP carried out the molecular genetic studies; $\mathrm{DP}, \mathrm{KM}, \mathrm{HJ}$ and SN analyzed data; DP and JJ wrote the paper. All authors read and approved the final manuscript.

\section{Competing interests}

The authors declare that they have no competing interests.

Received: 25 March 2011 Accepted: 18 August 2011

Published: 18 August 2011

\section{References}

1. Parker SL, Tong T, Bolden S, Winggo PA: Cancer statistics. CA Cancer J Clin 1997, 65:5-27.

2. Sakuragi N, Hirai A, Tada M, Yamada H, Yamamoto R, Fujimoto S, Moriuchi T: Dominant-negative mutation of p53 tumor suppressor gene in endometrial carcinoma. Gynecol Oncol 2001, 83:485-490.

3. Kraljevic Pavelic S, Sedic M, Bosnjak H, Spaventi S, Pavelic K: Metastasis: new perspectives on an old problem. Mol Cancer 2011, 10:22.

4. Vonlanthen S, Heighway J, Altermatt HJ, Gugger M, Kappeler A, Borner MM, van Lohuizen M, Betticher DC: The bmi-1 oncoprotein is differentially expressed in nonsmall cell lung cancer and correlates with INK4A-ARF locus expression. Br J Cancer 2001, 84:1372-6.

5. Guo BH, Feng Y, Zhang R, Xu LH, Li MZ, Kung HF, Song LB, Zeng MS: Bmi1 promotes invasion and metastasis, and its elevated expression is correlated with an advanced stage of breast cancer. Mol Cancer 2011, 10(1):10..

6. Glinsky GV, Berezovska O, Glinskii AB: Microarray analysis identifies a death-from-cancer signature predicting therapy failure in patients with multiple types of cancer. J Clin Invest 2005, 115:1503-21.

7. Zhang F, Sui L, Xin T: Correlations of BMI-1 expression and telomerase activity in ovarian cancer tissues. Exp Oncol 2008, 30:70-4.

8. Honig A, Weidler C, Häusler S, Krockenberger M, Buchholz S, Köster F, Segerer SE, Dietl J, Engel JB: Overexpression of polycomb protein BMI-1 in human specimens of breast, ovarian, endometrial and cervical cancer. Anticancer Res 2010, 30:1559-64.

9. Sasaki M, Ikeda H, Itatsu K, Yamaguchi J, Sawada S, Minato H, Ohta T, Nakanuma $Y$ : The overexpression of polycomb group proteins Bmi1 and $\mathrm{EZH} 2$ is associated with the progression and aggressive biological behavior of hepatocellular carcinoma. Lab Invest 2008, 88:873-882.

10. Yang MH, Hsu DS, Wang HW, Wang HJ, Lan HY, Yang WH, Huang CH, Kao SY, Tzeng CH, Tai SK, Chang SY, Lee OK, Wu KJ: Bmi1 is essential in Twist1-induced epithelial-mesenchymal transition. Nat Cell Biol 2010, 12:982-92.

11. Tsai HC, Baylin SB: Cancer epigenetics: linking basic biology to clinical medicine. Cell Res 2011, 21(3):502-17.

12. Slaby O, Svoboda M, Michalek J, Vyzula R: MicroRNAs in colorectal cancer: translation of molecular biology into clinical application. Mol Cancer 2009, 8:102.

13. Castilla MÁ, Moreno-Bueno G, Romero-Pérez L, Van De Vijver K, Biscuola M, López-García MÁ, Prat J, Matías-Guiu X, Cano A, Oliva E, Palacios J: MicroRNA signature of the epithelial-mesenchymal transition in endometrial carcinosarcoma. J Pathol 2011, 223:72-80.

14. Tie J, Pan Y, Zhao L, Wu K, Liu J, Sun S, Guo X, Wang B, Gang Y, Zhang Y, Li Q, Qiao T, Zhao Q, Nie Y, Fan D: MiR-218 Inhibits Invasion and Metastasis of Gastric Cancer by Targeting the Robo1 Receptor. PLoS Genet 2010, 6:e1000879.

15. Chen $R$, Alvero AB, Silasi DA, Kelly MG, Fest $S$, Visintin I, Leiser $A$, Schwartz PE, Rutherford T, Mor G: Regulation of IKKbeta by miR-199a affects NF-kappaB activity in ovarian cancer cells. Oncogene 2008, 27:4712-23.

16. Xia H, Qi Y, Ng SS, Chen X, Li D, Chen S, Ge R, Jiang S, Li G, Chen Y, He ML Kung HF, Lai L, Lin MC: MicroRNA-146b inhibits glioma cell migration and invasion by targeting MMPs. Brain Res 2009, 1269:158-65.

17. Dong $P, X u Z$, Jia N, Li D, Feng Y: Elevated expression of p53 gain-offunction mutation $\mathrm{R} 175 \mathrm{H}$ in endometrial cancer cells can increase the invasive phenotypes by activation of the EGFR/PI3K/AKT pathway. Mol Cancer 2009, 8:103-110.

18. Yu CC, Lo WL, Chen YW, Huang PI, Hsu HS, Tseng LM, Hung SC, Kao SY, Chang CJ, Chiou SH: Bmi-1 regulates Snail expression and promotes metastasis ability in head and neck squamous cancer-derived ALDH1 positive cells. J Oncol 2011, 2011, pii: 609259.

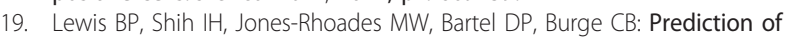
mammalian microRNA targets. Cell 2003, 115(7):787-98.

20. VGodlewski J, Nowicki MO, Bronisz A, Williams S, Otsuki A, Nuovo G, Raychaudhury A, Newton HB, Chiocca EA, Lawler S: Targeting of the Bmi-1 
Oncogene/Stem Cell Renewal Factor by MicroRNA-128 Inhibits Glioma Proliferation and Self-Renewal. Cancer Res 2008, 68(22):9125-30.

21. Jagani Z, Wiederschain D, Loo A, He D, Mosher R, Fordjour P, Monahan J, Morrissey M, Yao YM, Lengauer C, Warmuth M, Sellers WR, Dorsch M: The Polycomb group protein Bmi-1 is essential for the growth of multiple myeloma cells. Cancer Res 2010, 70:5528-38.

22. Jacobs JJ, Kieboom K, Marino S, DePinho RA, van Lohuizen M: The oncogene and Polycomb-group gene bmi-1 regulates cell proliferation and senescence through the ink4a locus. Nature 1999, 397:164-8.

23. Hoenerhoff MJ, Chu I, Barkan D, Liu ZY, Datta S, Dimri GP, Green JE: BMI1 cooperates with H-RAS to induce an aggressive breast cancer phenotype with brain metastases. Oncogene 2009, 28:3022-32.

24. Jiang Y, Su B, Meng X, Liu C, Liu B, Liu D, Fan Y, Yang H: Effect of siRNAmediated silencing of Bmi-1 gene expression on HeLa cells. Cancer Sci 2010, 101:379-86.

25. Song LB, Li J, Liao $W T$, Feng $Y$, Yu CP, Hu LJ, Kong QL, Xu LH, Zhang $X$, Liu WL, Li MZ, Zhang L, Kang TB, Fu LW, Huang WL, Xia YF, Tsao SW, Li M, Band $V$, Band $H$, Shi QH, Zeng YX, Zeng MS: The polycomb group protein Bmi-1 represses the tumor suppressor PTEN and induces epithelialmesenchymal transition in human nasopharyngeal epithelial cells. $J$ Clin Invest 2009, 119:3626-36.

26. Gregory PA, Bracken CP, Bert AG, Goodall GJ: MicroRNAs as regulators of epithelial-mesenchymal transition. Cell Cycle 2008, 7:3112-8.

27. Gregory PA, Bert AG, Paterson EL, Barry SC, Tsykin A, Farshid G, Vadas MA, Khew-Goodall Y, Goodall GJ: The miR-200 family and miR-205 regulate epithelial to mesenchymal transition by targeting ZEB1 and SIP1. Nat Cell Biol 2008, 10:593-601.

28. Meng Z, Fu X, Chen X, Zeng S, Tian Y, Jove R, Xu R, Huang W: MiR-194 is a marker of hepatic epithelial cells and suppresses metastasis of liver cancer cells in mice. Hepatology 2010, 52:2148-57.

29. Liu G, Yuan X, Zeng Z, Tunici P, Ng H, Abdulkadir IR, Lu L, Irvin D, Black KL, Yu JS: Analysis of gene expression and chemoresistance of CD133+ cancer stem cells in glioblastoma. Mol Cancer 2006, 5:67.

30. Hubbard SA, Friel AM, Kumar B, Zhang L, Rueda BR, Gargett CE: Evidence for cancer stem cells in human endometrial carcinoma. Cancer Res 2009, 69:8241-8.

doi:10.1186/1476-4598-10-99

Cite this article as: Dong et al:: MicroRNA-194 inhibits epithelial to mesenchymal transition of endometrial cancer cells by targeting oncogene BMI-1. Molecular Cancer 2011 10:99.

\section{Submit your next manuscript to BioMed Central and take full advantage of:}

- Convenient online submission

- Thorough peer review

- No space constraints or color figure charges

- Immediate publication on acceptance

- Inclusion in PubMed, CAS, Scopus and Google Scholar

- Research which is freely available for redistribution

Submit your manuscript at www.biomedcentral.com/submit 